\title{
Comparative analysis of zinc-blende and wurtzite GaN for full-band polar optical phonon scattering and negative differential conductivity
}

C. Bulutay, B. K. Ridley, and N. A. Zakhleniuk

Citation: Appl. Phys. Lett. 77, 2707 (2000); doi: 10.1063/1.1320020

View online: https://doi.org/10.1063/1.1320020

View Table of Contents: http://aip.scitation.org/toc/apl/77/17

Published by the American Institute of Physics

\section{Articles you may be interested in}

Transition between wurtzite and zinc-blende GaN: An effect of deposition condition of molecular-beam epitaxy Applied Physics Letters 89, 151921 (2006); 10.1063/1.2360916

Comparison of zinc-blende and wurtzite GaN semiconductors with spontaneous polarization and piezoelectric field effects

Journal of Applied Physics 87, 353 (2000); 10.1063/1.371915

Electronic transport studies of bulk zincblende and wurtzite phases of GaN based on an ensemble Monte Carlo calculation including a full zone band structure

Journal of Applied Physics 78, 1033 (1995); 10.1063/1.360405

Electronic band structures and effective-mass parameters of wurtzite $\mathrm{GaN}$ and InN

Journal of Applied Physics 83, 1429 (1998); 10.1063/1.366847

Band gap engineering of wurtzite and zinc-blende GaN/AIN superlattices from first principles Journal of Applied Physics 108, 103701 (2010); 10.1063/1.3505752

Deep traps in AIGaN/GaN heterostructures studied by deep level transient spectroscopy: Effect of carbon concentration in GaN buffer layers

Journal of Applied Physics 108, 063706 (2010); 10.1063/1.3488610

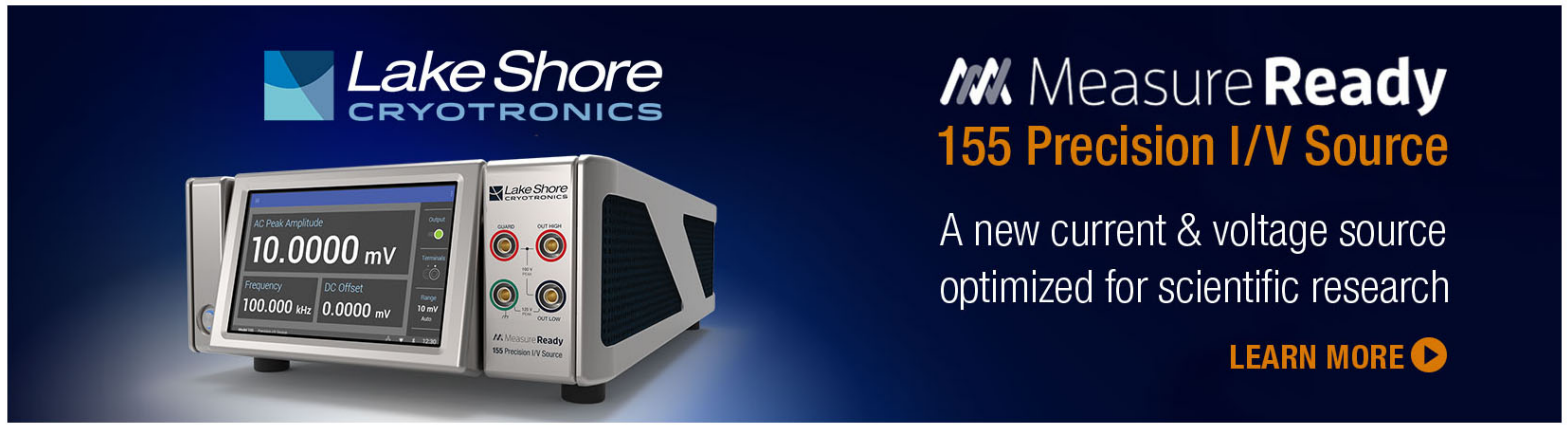




\title{
Comparative analysis of zinc-blende and wurtzite GaN for full-band polar optical phonon scattering and negative differential conductivity
}

\author{
C. Bulutay ${ }^{\mathrm{a})}$ and B. K. Ridley \\ Department of Electronic Systems Engineering, University of Essex, Colchester C04 3SQ, United Kingdom \\ N. A. Zakhleniuk \\ Caswell Technology, Marconi Caswell, Towcester, Northants, NN12 8EQ, United Kingdom
}

(Received 26 May 2000; accepted for publication 1 September 2000)

\begin{abstract}
For high-power electronics applications, $\mathrm{GaN}$ is a promising semiconductor. Under high electric fields, electrons can reach very high energies where polar optical phonon (POP) emission is the dominant scattering mechanism. So, we undertake a full-band analysis of POP scattering of conduction-band electrons based on an empirical pseudopotential band structure. To uncover the directional variations, we compute POP emission rates along high-symmetry directions for the zinc-blende (ZB) crystal phase of GaN. We also compare the results with those of the wurtzite phase. In general, the POP scattering rates in the zinc-blende phase are lower than the wurtzite phase. Our analysis also reveals appreciable directional dependence, with the $\Gamma-L$ direction of $\mathrm{ZB}$ $\mathrm{GaN}$ being least vulnerable to POP scattering, characterized by a scattering time of $11 \mathrm{fs}$. For both crystal phases, we consider the negative differential conductivity possibilities driven by the negative effective mass part of the band structure. According to our estimation, for the ZB phase the onset of this effect requires fields above $\sim 1 \mathrm{MV} / \mathrm{cm}$. (C) 2000 American Institute of Physics.
\end{abstract}

[S0003-6951(00)02743-1]

The wide-band-gap III-nitride semiconductors are currently being considered for high-power and high-temperature applications. Among these III nitrides, the most studied is GaN which can be grown either in wurtzite (WZ) or zincblende (ZB) phases depending on the choice of the substrate and the growth conditions. ${ }^{1}$ The mounting interest in the high-field applications of GaN, brings under demand a detailed knowledge of the transport properties, like the characterization of the dominant scattering mechanism. This, in $\mathrm{GaN}$ is the polar optical phonon (POP) scattering due to its high degree of ionicity. ${ }^{2}$ A realistic high-field analysis inevitably requires a full-band treatment as the carriers under the influence of such high fields move further away from the band edge where their scattering rate depends on the details of the band structure.

In this letter, we present a full-band analysis of the POP scattering of conduction-band (CB) electrons based on the empirical pseudopotential band structure. To shed light on the effects of the crystal phase, we consider both ZB and WZ structures. We place more emphasis on the $\mathrm{ZB}$ phase, as a comprehensive discussion of the $\mathrm{WZ}$ case has been given by us elsewhere. ${ }^{3}$ For the empirical pseudopotential band structure of $\mathrm{ZB} \mathrm{GaN}$, we use published form factors, ${ }^{4}$ however, for the case of WZ GaN we felt the necessity to develop our own form factors ${ }^{3}$ due to the poor performance of the $\mathrm{CB}$ properties of the available ones.

Considering the $\mathrm{ZB}$ case, the scattering rate based on Fermi's Golden Rule due to POP emission of an electron at the band $m$, with a wave vector $\mathbf{k}$, is given by

\footnotetext{
a) Author to whom correspondence should be addressed; present address: Department of Physics, Bilkent University, 06533 Ankara, Turkey; electronic-mail: bulutay@fen.bilkent.edu.tr
}

$$
\begin{aligned}
W_{m}(\mathbf{k})= & \frac{2 \pi}{\hbar} \frac{V}{(2 \pi)^{3}} \sum_{m^{\prime}} \int_{1 s t B Z} d^{3} k^{\prime} \Delta_{m^{\prime}, m}\left(\mathbf{k}^{\prime}, \mathbf{k}\right) \\
& \times\left|C_{\mathrm{POP}}(q)\right|^{2} \delta\left(E_{m^{\prime}}\left(\mathbf{k}^{\prime}\right)-E_{m}(\mathbf{k})+\hbar \omega_{\mathrm{LO}}\right),
\end{aligned}
$$

where the emitted phonon wave vector is $\mathbf{q}=-\mathbf{k}^{\prime}+\mathbf{k}$ mapped to first $\mathrm{BZ}$, the primed indices represent final-state electron labels over which a summation/integration is performed, $V$ is the total crystal volume, $E_{m}(\mathbf{k})$ is the band energy of the electron, and $\hbar \omega_{\mathrm{LO}}$ is the longitudinal-optical (LO) phonon energy assumed to be dispersionless. The cubic POP coupling constant is given by $\left|C_{\mathrm{POP}}(q)\right|^{2}$ $=2 \pi e^{2} \hbar \omega_{\mathrm{LO}}\left(\epsilon_{\infty}^{-1}-\epsilon_{0}^{-1}\right) /\left(V q^{2}\right)$, where $\epsilon_{0}$ and $\epsilon_{\infty}$ are the static and high-frequency dielectric constants. The cellperiodic overlap parameter is given by $\Delta_{m^{\prime}, m}\left(\mathbf{k}^{\prime}, \mathbf{k}\right)$ $=\left|(1 / \Omega) \int_{\Omega} u_{m^{\prime}, \mathbf{k}^{\prime}}^{*}(\mathbf{r}) u_{m, \mathbf{k}}(\mathbf{r}) d^{3} r\right|^{2}$, where $u_{m, \mathbf{k}}(\mathbf{r})$ is the cellperiodic part of the Bloch wave function and $\Omega$ is the volume of the primitive cell. This overlap parameter is vital to account for the restrictions brought by the symmetries of the participating wave functions to the scattering probabilities. For the parameters of GaN, up to room temperature the thermal phonon occupation remains negligible, hence, Eq. (1) actually governs the overall scattering rate up to room temperature.

We compute the scattering rate in two alternative ways: ${ }^{3}$ by direct integration over two spatial variables using the delta function or by means of the Lehmann-Taut BZ integration technique. ${ }^{5}$ In the former, we prefer to work in spherical coordinates and enclose the truncated-octahedronshaped first Brillouin zone (BZ) by a sphere of radius $(\sqrt{5 / 2})(2 \pi / a)$, where $a$ is the lattice constant. The regions of the sphere that lie outside the first BZ are discarded by introducing a unit step function into the integrand. We em- 

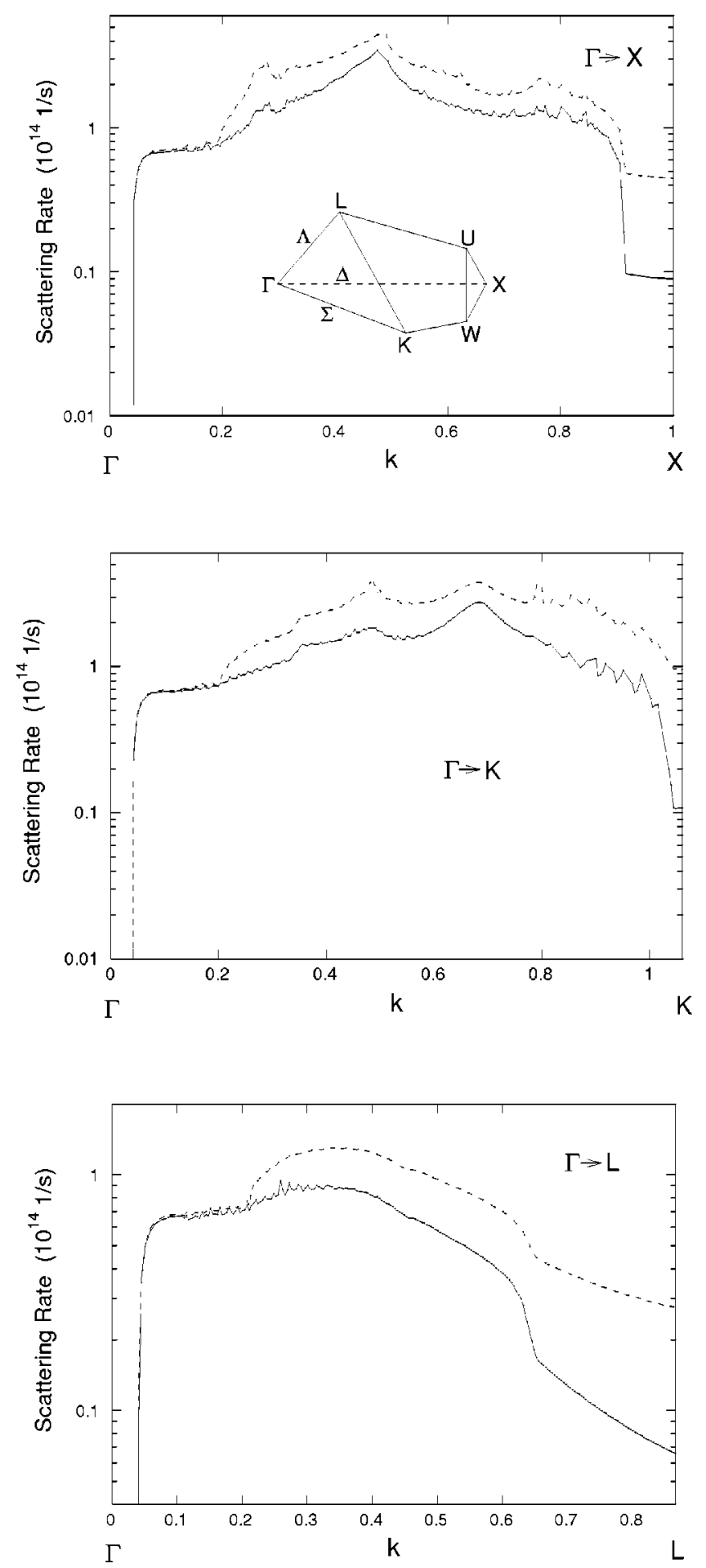

FIG. 1. POP scattering rate vs wave vector (in units of $2 \pi / a$ ) along the directions $\Gamma-X, \Gamma-K$, and $\Gamma-L$. The dashed lines indicate the same rates when the cell-periodic overlaps are taken to be unity. Inset in the upper figure shows the high-symmetry points and directions in the ZB irreducible wedge.

ploy the linear tetrahedron interpolation ${ }^{5}$ of band energies and store the $\mathrm{CB}$ energies by sampling the irreducible wedge of the BZ by 16114 data points, giving more emphasis to the vicinity of the $\Gamma$ point. For the cell-periodic Bloch overlap parameter $\Delta_{m^{\prime}, m}$, we use a less dense sampling of the irreducible wedge by 1604 final-state data points for each initial electron state under consideration.

To be consistent with our previous WZ GaN analysis, ${ }^{3}$ we use the following data for the cubic $\mathrm{GaN}$ phonons:

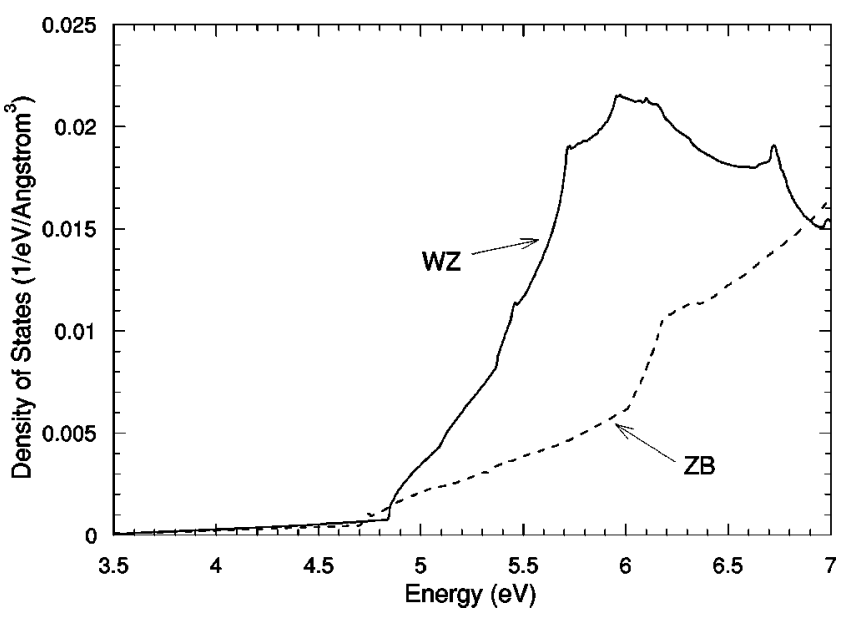

FIG. 2. Density of states per spin, per volume of the ZB (dashed) and WZ (solid) phases of $\mathrm{GaN}$ for the lowest conduction-band energies. The zero level of the energy is set to valence-band maximum.

$\hbar \omega_{\mathrm{LO}}=92.12 \mathrm{meV}, \epsilon_{0}=9.28$, and $\epsilon_{\infty}=5.29$. Aiming for a directional assessment, we start from the $\mathrm{CB}$ minimum at the $\Gamma$ point, and trace the POP scattering rate of $\mathrm{CB}$ electrons along high-symmetry lines: $\Delta, \Sigma, \Lambda$ corresponding to $\Gamma-X$, $\Gamma-K$, and $\Gamma-L$ directions, respectively; see the inset in Fig. 1. The effect of setting the cell-periodic overlap parameter to unity is indicated by the dashed lines in Fig. 1 for each direction; note the resultant overestimation in the scattering rates away from the $\mathrm{CB}$ edge. The results indicate that the $\Gamma-X(\Gamma-L)$ direction has the highest (lowest) scattering rate. This directional dependence can be attributed to the $\mathrm{CB}$ satellite valley being located at the $X$ point, which is the major scattering destination at high energies, providing high density of final states. As the $\Gamma-L$ direction is further away from the $X$ valley, it requires relatively larger phonon-wave vectors, which leads to less electron-phonon coupling. Furthermore, the lowest $\mathrm{CB}$ along the $\Gamma-L$ direction ${ }^{4}$ does not enable an intravalley POP emission to the $L$ point, which is the key factor behind the low scattering rate.

The $\Gamma-L$ directions of $\mathrm{ZB} \mathrm{GaN}$ are related to the $\Gamma-A$ and $\Gamma-U$ directions of $\mathrm{WZ} \mathrm{GaN} .{ }^{6}$ We further note that in

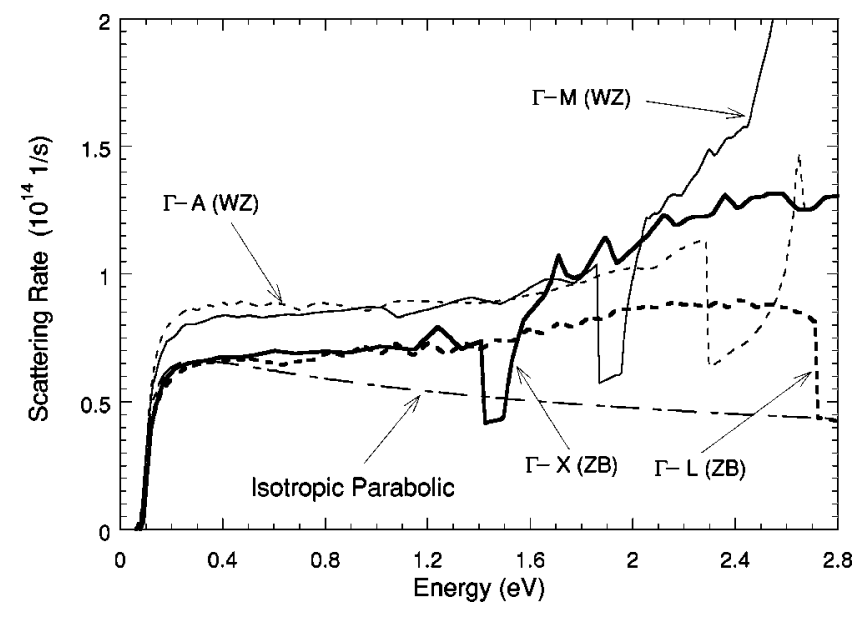

FIG. 3. POP scattering rate vs energy, comparing the $\mathrm{ZB}$ rates with the $\mathrm{WZ}$ LO-like rates along several directions. To aid comparison, for both crystal phases the energy reference is chosen to be at a same CB minimum. Cubic isotropic parabolic POP rate calculated using the $\mathrm{CB}$ edge effective mass of the $\mathrm{ZB}$ phase is also included. 


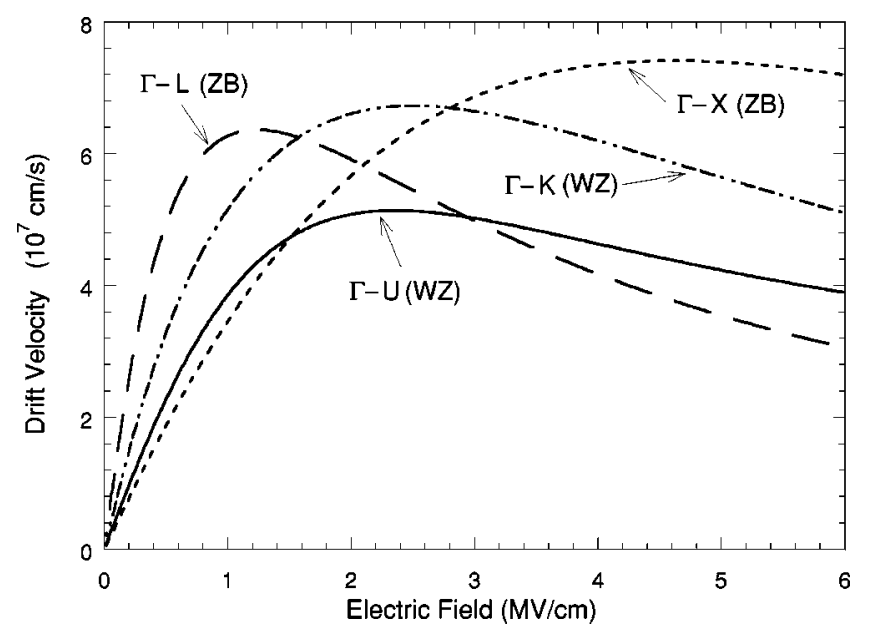

FIG. 4. Comparison of drift velocity vs electric field for the ZB and WZ phases along several directions.

WZ GaN, there exists a $\mathrm{BZ}$ folding along the $c$ axis of the crystal, therefore, for the $\Gamma-A$ (WZ) direction both the lowest $\mathrm{CB}$ and its folded extension need to be considered. ${ }^{3}$ In general terms, the ZB phase has a smaller POP scattering rate than the WZ phase, which is essentially due to the lower density of states in the former. The quantitative comparison of the density of states for these two phases around the energies relevant to our work is given in Fig. 2. However, the lower density of states in $\mathrm{ZB} \mathrm{GaN}$ is shown to have one adverse consequence by leading to a lower breakdown voltage in the $\mathrm{ZB}$ phase due to easier heating of the $\mathrm{CB}$ electrons under high fields, provoking impact ionization. ${ }^{7}$ The effect of the $M$ and $X$ satellite valleys $\left(E_{\mathrm{M}}=1.87 \mathrm{eV}, E_{\mathrm{X}}=1.41 \mathrm{eV}\right.$ above the $\mathrm{CB}$ edge) along the $\Gamma-M(\mathrm{WZ})$ and $\Gamma-X(\mathrm{ZB})$ directions, respectively, are seen in Fig. 3. Just at these valley energies the average scattering rate suddenly drops as electrons at the bottom of this valley contribute very little to the POP scattering rate, limited to intervalley scattering with large phonon-wave vectors. However, when the energy is increased further to allow the electrons in these valleys to have intravalley scattering, the rate rapidly rises. The isotropic-parabolic band estimation for the POP rate is also shown to illustrate the validity range of this approximation as compared to a full-band treatment; only up to $\sim 0.4 \mathrm{eV}$ above the $\mathrm{CB}$ edge there is seen to be a good agreement.

Following Esaki and Tsu's phenomenological semiclassical treatment, ${ }^{8}$ we estimate the drift velocity dependence on the electric field. Accordingly, the average drift velocity assuming a constant scattering time $\tau$ is given by

$$
v_{d}=e F \hbar^{-2} \int_{0}^{\infty} \frac{\partial^{2} E_{\mathrm{Fld}}}{\partial k_{\mathrm{Fld}}^{2}} e^{-t / \tau} d t,
$$

where $\partial^{2} E_{\mathrm{Fld}} / \partial k_{\mathrm{Fld}}^{2}$ is the curvature of the energy-band diagram along the applied field's direction $\mathbf{F}$, sampled at the $\mathbf{k}$ point, $k_{\mathrm{Fld}}(t)=e F t / \hbar$, for an electron originating from the $\Gamma$ point. For the scattering time $\tau$ in Eq. (2), we use the value given by the maximum scattering rate in each direction. Figure 4 shows the corresponding drift velocity estimations relevant to high fields for both crystal phases. Different low- field mobilities in Fig. 4, contrary to reality, are caused by using the maximum scattering rate throughout the band, even though this rate shows an isotropic character close to the $\mathrm{CB}$ edge (cf. Fig. 1). The $\Gamma-L$ direction of $\mathrm{ZB} \mathrm{GaN}$ has the lowest onset field of $1 \mathrm{MV} / \mathrm{cm}$ for the observation of negative differential conductivity (NDC), however, note the sensitivity of the onset field to angular variations (cf. Fig. 4). There have already been several predictions of NDC in GaN (Refs. 9-12) at field levels of around $150 \mathrm{kV} / \mathrm{cm}$, all being based on the transfer of electrons to upper valleys, whereas, the NDC that we refer to here is caused by those carriers reaching the negative effective mass part of the band structure at high fields before undergoing a scattering event, an idea initially proposed by Krömer. ${ }^{13}$ Recently, the possibility of NDC based on this mechanism was advocated ${ }^{14}$ for cubic $\mathrm{GaN}$ beyond a field of $\sim 50 \mathrm{kV} / \mathrm{cm}$. This onset voltage is about 20 times lower than our estimate. Recent Monte Carlo investigations ${ }^{15}$ along this line did not encounter any drastic change up to fields $\sim 0.5 \mathrm{MV} / \mathrm{cm}$ with respect to a variation of the energy of the $X$ valley, which further contradicts this previous prediction. ${ }^{14}$

In summary, to aid the high-field characterization of GaN, we present a full-band POP scattering analysis of $\mathrm{CB}$ electrons, comparing the $\mathrm{ZB}$ and $\mathrm{WZ}$ crystal phases. In general, the scattering rates in the $\mathrm{ZB}$ phase are lower than the WZ phase due to the lower density of states in the former. This suggests a possibility to have enhanced high-field mobility in $\mathrm{ZB} \mathrm{GaN}$ in comparison with $\mathrm{WZ}$ GaN. High-field transport along the $\Gamma-L$ direction of $\mathrm{ZB}$ GaN characterized by a scattering time of $11 \mathrm{fs}$ has the lowest scattering rate, as the POP emission to the $L$ point is energetically not possible. The observation of NDC driven by the negative effective mass part of the band structure is predicted beyond a field of roughly $1 \mathrm{MV} / \mathrm{cm}$ for the $\mathrm{ZB}$ phase, and $2.3 \mathrm{MV} / \mathrm{cm}$ for the WZ phase.

This work is supported by ONR (Contract No. N0001499-1-0014) and EPSRC (Contract No. GR/L/56725).

${ }^{1}$ J. W. Orton and C. T. Foxon, Rep. Prog. Phys. 61, 1 (1998).

${ }^{2}$ A. García and M. L. Cohen, Phys. Rev. B 47, 4215 (1993); 47, 4221 (1993).

${ }^{3}$ C. Bulutay, B. K. Ridley, and N. A. Zakhleniuk, Phys. Rev. B (in press).

${ }^{4}$ R. Wang, P. P. Ruden, J. Kolnik, I. Oğuzman, and K. F. Brennan, J. Phys. Chem. Solids 58, 913 (1997).

${ }^{5}$ G. Lehmann and M. Taut, Phys. Status Solidi B 54, 469 (1972).

${ }^{6}$ M. R. Salehpour and S. Satpathy, Phys. Rev. B 41, 3048 (1990).

${ }^{7}$ M. Farahmand and K. F. Brennan, IEEE Trans. Electron Devices 47, 493 (2000).

${ }^{8}$ L. Esaki and R. Tsu, IBM J. Res. Dev. 14, 61 (1970).

${ }^{9}$ B. Gelmont, K. Kim, and M. Shur, J. Appl. Phys. 74, 1818 (1993).

${ }^{10}$ N. S. Mansour, K. W. Kim, and M. A. Littejohn, J. Appl. Phys. 77, 2834 (1995).

${ }^{11}$ J. Kolník, İ. H. Oğuzman, K. F. Brennan, R. Wang, P. P. Ruden, and Y. Wang, J. Appl. Phys. 78, 1033 (1995).

${ }^{12}$ B. E. Foutz, S. K. O'Leary, M. S. Shur, and L. F. Eastman, J. Appl. Phys. 85, 7727 (1999).

${ }^{13}$ H. Krömer, Phys. Rev. 109, 1856 (1958).

${ }^{14}$ S. Krishnamurthy, M. van Schilfgaarde, A. Sher, and A.-B. Chen, Appl. Phys. Lett. 71, 1999 (1997).

${ }^{15}$ K. F. Brennan (private communication). 